\title{
Ankündigungen
}

\section{Calls for Papers}

Crossing Borders in the History of Technology, ICOHTEC 35. Symposium 2008, 5.-10. August 2008, Victoria, British Columbia, Canada

Einsendeschluss: Early Decision for Proposals 14. Januar 2008; Final Deadline for Proposals 3. März 2008

Weitere Informationen: http://icohtec.uvic.ca/

The Culture of Print in Science, Technology, Engineering, and Medicine, The Center for the History of Print Culture in Modern America, 12.-13. September 2008, Madison, WI

Einsendeschluss: 15. Januar 2008

Weitere Informationen: http://slisweb.lis.wisc.edu/ printcul/conferences.html

From Semaphore to Cellular Radio Telecommunications, IEEE History of Technology Conference, HISTELCON 2008, 11.-12. September 2008, Paris Einsendeschluss: 31. Januar 2008

Weitere Informationen: http://www.ieee.org/web/aboutus/history_center/

Ideas and Instruments in Social Context, ICOHTEC 36. Symposium 2009, (Part of the XXIII International Congress of the History of Science and Technology), 26.-31. Juli 2009, Budapest

Einsendeschluss: 3. März 2008

Weitere Informationen: Reinhold Bauer (chair of the program committee), E-Mail: rbauer@hsu-hh.de

Shock Cities: Urban Form in Historical Perspective, Fourth Biennial Urban History Conference, 5.-8. November 2008, Houston, TX

Einsendeschluss: 3. März 2008

Weitere Informationen: http://uha.udayton.edu/html/conf.html

Third Annual „Science \& the Public“ Conference, CHSTM, University of Manchester, 21.-22. Juni 2008, Manchester, United Kingdom Einsendeschluss: 14. März 2008

Weitere Informationen: http://www.chstm.manchester.ac.uk/newsandevents/ conferences/scienceandthepublic/index.asp oder E-Mail: scienceandpublic@, googlemail.com 
Automobility: A Conference on the 100th anniversary of the Model T, Hagley Museum and Library, 6.-7. November 2008, Wilmington, DE

Einsendeschluss: 31. März 2008

Weitere Informationen: http://www.hagley.lib.de.us/events.html oder Carol Lockman (E-Mail: clockman@Hagley.org)

\section{Tagungen}

6.-9. Februar 2008, Zentrum für interdisziplinäre Forschung (ZiF), Universität Bielefeld: IMAGINING OUTER SPACE, 1900-2000, Bielefeld Weitere Informationen: http://www.geschkult.fu-berlin.de/e/fmi/arbeitsbereiche/ab_nolte/Bielefeld/Hauptseite.html

7.-8. Februar 2008, Technikgeschichtliche Jahrestagung des VDI: Sport und Technik, Düsseldorf

Weitere Informationen: http://www.vdi.de/vdi/organisation/schnellauswahl/ hauptgruppe/gesellschaft/10111/

21.-23. Februar 2008, Deutsches Historisches Institut, Washington, DC: Managing the Unknown: Natural Reserves in Historical Perspective, Washington, DC

Weitere Informationen: http://www.ghi-dc.org/events/upcoming.html

14. März 2008, University of California, Santa Barbara: Science and Technology, 1500-1800, Santa Barbara, CA

Weitere Informationen: http://emc.english.ucsb.edu/conferences/2007-2008/ Science\&Technology 1500-1800/

4.-6. April 2008, 26. MEPHISTOS Graduate Student Conference: History, Philosophy, Sociology and Anthropology of Science, Technology, and Medicine, Austin, TX

Weitere Informationen: http://studentorgs.utexas.edu/mephistos/

\section{Stipendien}

Bakken Library Research Programs, Minneapolis, MN: Visiting Research Fellowships und Travel Grants für 2008

Bewerbungsschluss: Visiting Research Fellowships: 15. Februar 2008; Travel Grants: laufend

Weitere Informationen: http://www.thebakken.org/research/research.htm\# BakkenFellowshipsandGrants 